\title{
Two Newton methods on the manifold of fixed-rank matrices endowed with Riemannian quotient geometries *
}

\author{
P.-A. Absil ${ }^{\dagger} \quad$ Luca Amodei ${ }^{\ddagger} \quad$ Gilles Meyer ${ }^{\S}$
}

October 17, 2018

\begin{abstract}
We consider two Riemannian geometries for the manifold $\mathcal{M}(p, m \times n)$ of all $m \times n$ matrices of rank $p$. The geometries are induced on $\mathcal{M}(p, m \times n)$ by viewing it as the base manifold of the submersion $\pi:(M, N) \mapsto M N^{\mathrm{T}}$, selecting an adequate Riemannian metric on the total space, and turning $\pi$ into a Riemannian submersion. The theory of Riemannian submersions, an important tool in Riemannian geometry, makes it possible to obtain expressions for fundamental geometric objects on $\mathcal{M}(p, m \times n)$ and to formulate the Riemannian Newton methods on $\mathcal{M}(p, m \times n)$ induced by these two geometries. The Riemannian Newton methods admit a stronger and more streamlined convergence analysis than the Euclidean counterpart, and the computational overhead due to the Riemannian geometric machinery is shown to be mild. Potential applications include low-rank matrix completion and other low-rank matrix approximation problems.
\end{abstract}

Key words. fixed-rank matrices; manifold; differential geometry; Riemannian geometry; Riemannian submersion; Levi-Civita connection; Riemannian connection; Riemannian exponential map; geodesics; Newton's method

\section{Introduction}

Let $m, n$, and $p \leq \min \{m, n\}$ be positive integers and let $\mathcal{M}(p, m \times n)$ denote the set of all rank- $p$ matrices of size $m \times n$,

$$
\mathcal{M}(p, m \times n)=\left\{X \in \mathbb{R}^{m \times n}: \operatorname{rank}(X)=p\right\} .
$$

${ }^{*}$ This paper presents research results of the Belgian Network DYSCO (Dynamical Systems, Control, and Optimization), funded by the Interuniversity Attraction Poles Programme, initiated by the Belgian State, Science Policy Office. The scientific responsibility rests with its authors.

${ }^{\dagger}$ Department of Mathematical Engineering, ICTEAM Institute, Université catholique de Louvain, B-1348 Louvain-la-Neuve, Belgium (http://sites.uclouvain.be/absil/).

${ }^{\ddagger}$ Institut de Mathmatiques de Toulouse, Universit Paul Sabatier, 118 route de Narbonne, 31062 Toulouse cedex 9, France (luca.amodei@math.univ-toulouse.fr).

${ }^{\S}$ Department of Electrical Engineering and Computer Science, University of Liège, B-4000 Liège, Belgium (gillesmy@gmail.com). 
Given a smooth function $f: \mathcal{M}(p, m \times n) \rightarrow \mathbb{R}$, we consider the problem

$$
\min f(X) \text { subject to } X \in \mathcal{M}(p, m \times n) \text {. }
$$

Problem (21) subsumes low-rank matrix approximation problems, where $f(X) \equiv\|A-X\|^{2}$ with $A \in \mathbb{R}^{m \times n}$ given and $\|\cdot\|$ a (semi)norm. In particular, it includes low-rank matrix completion problems, which have been the topic of much attention recently; see KMO10, DMK11, BA11, Van11, MMBS11, DKM12] and references therein. Interestingly, low-rank matrix completion problems combine two sparsity aspects: only a few elements of $A$ are available, and the vector of singular values of $X$ is restricted to have only a few nonzero elements.

This paper belongs to a trend of research, see HM94, HS95, SE10, Van11, MMS11, MMBS11, where problem (2) is tackled using differential-geometric techniques exploiting the fact that $\mathcal{M}(p, m \times n)$ is a submanifold of $\mathbb{R}^{m \times n}$. We are interested in Riemannian Newton methods (see $\underline{\mathrm{Smi} 94}, \mathrm{ADM}^{+} 02$, $\mathrm{AMS08}$ ) for problem (2), with a preference for the pure Riemannian setting Smi94]. This setting involves defining a Riemannian metric on $\mathcal{M}(p, m \times n)$ and providing an expression for the Riemannian connection - which underlies the Riemannian Hessian - and for the Riemannian exponential. When $\mathcal{M}(p, m \times n)$ is viewed as a Riemannian submanifold of $\mathbb{R}^{m \times n}$, the necessary ingredients for computing the Riemannian Hessian are available [Van11, §2.3], but a closed-form expression of the Riemannian exponential has been elusive in that geometry.

In this paper, we follow a different approach that strongly relies on two-term factorizations of low-rank matrices. To this end, let

$$
\mathbb{R}_{*}^{m \times p}=\left\{X \in \mathbb{R}^{m \times p}: \operatorname{rank}(X)=p\right\}
$$

denote the set of all full-rank $m \times p$ matrices, and observe that, since the function

$$
\pi: \mathbb{R}_{*}^{m \times p} \times \mathbb{R}_{*}^{n \times p} \rightarrow \mathcal{M}(p, m \times n):(M, N) \mapsto M N^{\mathrm{T}}
$$

is surjective, problem (2) amounts to the optimization over its domain of the function $\bar{f}=f \circ \pi$, i.e.,

$$
\bar{f}: \mathbb{R}_{*}^{m \times p} \times \mathbb{R}_{*}^{n \times p} \rightarrow \mathbb{R}:(M, N) \mapsto f\left(M N^{\mathrm{T}}\right) .
$$

Pleasantly, whereas $\mathcal{M}(p, m \times n)$ is a nonlinear space, $\mathbb{R}_{*}^{m \times p} \times \mathbb{R}_{*}^{n \times p}$ is an open subset of a linear space; more precisely, $\mathbb{R}_{*}^{m \times p} \times \mathbb{R}_{*}^{n \times p}$ is the linear space $\mathbb{R}^{m \times p} \times \mathbb{R}^{n \times p}$ with a nowhere dense set excerpted. The downside is that the minimizers of $\bar{f}$ are never isolated; indeed, for all $(M, N) \in \mathbb{R}_{*}^{m \times p} \times \mathbb{R}_{*}^{n \times p}, \bar{f}=f \circ \pi$ assumes the same value $\bar{f}(M, N)$ at all points of

$$
\pi^{-1}\left(M N^{\mathrm{T}}\right)=\left\{\left(M R, N R^{-\mathrm{T}}\right): R \in \mathrm{GL}(p)\right\},
$$

where

$$
\mathrm{GL}(p)=\left\{R \in \mathbb{R}^{p \times p}: \operatorname{det}(R) \neq 0\right\}
$$

denotes the general linear group of degree $p$. In the context of Newton-type methods, this can be a source of concern since, whereas the convergence theory of Newton's method to nondegenerate minimizers is well understood (see, e.g., [DS83, Theorem 5.2.1]), the situation becomes more intricate in the presence of non-isolated minimizers (see, e.g., GR85]).

The proposed remedy to this downside consists in elaborating a Riemannian Newton method that evolves conceptually on $\mathcal{M}(p, m \times n)$-avoiding the structural degeneracy in 
$\mathbb{R}_{*}^{m \times p} \times \mathbb{R}_{*}^{n \times p}$ —while still being formulated in $\mathbb{R}_{*}^{m \times p} \times \mathbb{R}_{*}^{n \times p}$. This is made possible by endowing $\mathbb{R}_{*}^{m \times p} \times \mathbb{R}_{*}^{n \times p}$ and $\mathcal{M}(p, m \times n)$ with Riemannian metrics that turn $\pi$ into a Riemannian submersion. The theory of Riemannian submersions ['N66, O'N83, then provides a way of representing the Riemannian connection and the Riemannian exponential of $\mathcal{M}(p, m \times n)$ in terms of the same objects of $\mathbb{R}_{*}^{m \times p} \times \mathbb{R}_{*}^{n \times p}$.

It should be pointed out that the local quadratic convergence of the Riemannian Newton method is retained if the Riemannian connection is replaced by any affine connection and the Riemannian exponential is replaced by any first-order approximation, termed retraction; see [AMS08, §6.3]. The preference for the pure Riemannian setting is thus mainly motivated by the mathematical elegance of a method fully determined by the sole Riemannian metric.

Some of the material of this paper is inspired from the PhD thesis Mey11 and the talk ADY09].

The paper is organized as follows. In the short sections 2 and 3 , we show that $\pi$ is a submersion and we recall some fundamentals of Riemannian submersions. A first, natural but unsuccessful attempt at turning $\pi$ into a Riemannian submersion is presented in Section 4 . Two ways of achieving success are then presented in sections 5 and 6 . In Section 5 , the strategy consists of introducing a non-Euclidean Riemannian metric on $\mathbb{R}_{*}^{m \times p} \times \mathbb{R}_{*}^{n \times p}$, whereas in Section [6, the plan of action is to restrict $\mathbb{R}_{*}^{m \times p} \times \mathbb{R}_{*}^{n \times p}$ by imposing orthonormality of one of the factors. We obtain closed-form expressions for the Riemannian connection (in both cases) and for the Riemannian exponential (in the latter case). Conclusions are drawn in Section 7 .

\section{$2 \mathcal{M}(p, m \times n)$ as a quotient manifold}

The set $\mathcal{M}(p, m \times n)$ of rank- $p$ matrices of size $m \times n$ is known to be an embedded submanifold of dimension $p(m+n-p)$ of $\mathbb{R}^{m \times n}$, connected whenever $\max \{m, n\}>1$; see [HM94, Ch. 5, Prop. 1.14]. Hence $\pi$ (4) is a smooth surjective map between two manifolds.

We show that $\pi$ is a submersion, i.e., that the differential of $\pi$ is everywhere surjective. Observe that the tangent space to $\mathbb{R}_{*}^{m \times p} \times \mathbb{R}_{*}^{n \times p}$ at $(M, N)$ is given by

$$
\mathrm{T}_{(M, N)} \mathbb{R}_{*}^{m \times p} \times \mathbb{R}_{*}^{n \times p}=\mathbb{R}^{m \times p} \times \mathbb{R}^{n \times p} ;
$$

this comes from the fact that $\mathbb{R}_{*}^{m \times p} \times \mathbb{R}_{*}^{n \times p}$ is an open submanifold of the Euclidean space $\mathbb{R}^{m \times p} \times \mathbb{R}^{n \times p}$ AMS08, \$3.5.1]. For all $(M, N) \in \mathbb{R}_{*}^{m \times p} \times \mathbb{R}_{*}^{n \times p}$ and all $(\dot{M}, \dot{N}) \in \mathbb{R}^{m \times p} \times \mathbb{R}^{n \times p}$, we have $\mathrm{D} \pi(M, N)[(\dot{M}, \dot{N})]=\dot{M} N^{\mathrm{T}}+M \dot{N}^{\mathrm{T}}$. Working in a coordinate system where $M=\left[\begin{array}{ll}I & 0\end{array}\right]^{\mathrm{T}}$ and $N=\left[\begin{array}{ll}I & 0\end{array}\right]^{\mathrm{T}}$, one readily sees that the dimension of the range of the $\operatorname{map}(\dot{M}, \dot{N}) \mapsto \mathrm{D} \pi(M, N)[(\dot{M}, \dot{N})]$ is equal to $p(m+n-p)$, the dimension of the codomain of $\pi$. Hence $\pi$ is a submersion.

As a consequence, by the submersion theorem [AMS08, Proposition 3.3.3], the fibers $\pi^{-1}\left(M N^{\mathrm{T}}\right)$ are $p^{2}$-dimensional submanifolds of $\mathbb{R}_{*}^{m \times p} \times \mathbb{R}_{*}^{n \times p}$. Moreover, by [AMR88, Proposition 3.5.23], the equivalence relation $\sim$ on $\mathbb{R}_{*}^{m \times p} \times \mathbb{R}_{*}^{n \times p}$, defined by $\left(M_{a}, N_{a}\right) \sim\left(M_{b}, N_{b}\right)$ if and only if $\pi\left(M_{a}, N_{a}\right)=\pi\left(M_{b}, N_{b}\right)$, is regular and $\mathbb{R}_{*}^{m \times p} \times \mathbb{R}_{*}^{n \times p} / \sim$ is a quotient manifold diffeomorphic to $\mathcal{M}(p, m \times n)$.

\section{Riemannian submersion: principles}

Turning $\pi$ into a Riemannian submersion amounts to endowing its domain $\mathbb{R}_{*}^{m \times p} \times \mathbb{R}_{*}^{n \times p}$ with a Riemannian metric $\bar{g}$ that satisfies a certain invariance condition, described next. 
By definition, the vertical space $\mathcal{V}_{(M, N)}$ at a point $(M, N) \in \mathbb{R}_{*}^{m \times p} \times \mathbb{R}_{*}^{n \times p}$ is the tangent space to the fiber $\pi^{-1}\left(M N^{\mathrm{T}}\right)$ (6). We obtain

$$
\mathcal{V}_{(M, N)}=\left\{\left(M \dot{R},-N \dot{R}^{\mathrm{T}}\right): \dot{R} \in \mathbb{R}^{p \times p}\right\} .
$$

Let $\bar{g}$ be a Riemannian metric on $\mathbb{R}_{*}^{m \times p} \times \mathbb{R}_{*}^{n \times p}$. Then one defines the horizontal space $\mathcal{H}_{(M, N)}$ at $(M, N)$ to be the orthogonal complement of $\mathcal{V}_{(M, N)}$ in $\mathbb{R}^{m \times p} \times \mathbb{R}^{n \times p}$ relative to $\bar{g}_{(M, N)}$, i.e.,

$$
\mathcal{H}_{(M, N)}=\left\{(\dot{M}, \dot{N}) \in \mathbb{R}^{m \times p} \times \mathbb{R}^{n \times p}: \bar{g}_{(M, N)}\left((\dot{M}, \dot{N}),\left(M \dot{R},-N \dot{R}^{\mathrm{T}}\right)\right)=0, \forall \dot{R} \in \mathbb{R}^{p \times p}\right\} .
$$

Next, given a tangent vector $\dot{X}_{M N^{\mathrm{T}}} \in \mathrm{T}_{M N^{\mathrm{T}}} \mathcal{M}(p, m \times n)$, there is one and only one

$$
\dot{X}_{(M, N)} \in \mathcal{H}_{(M, N)} \quad \text { such that } \operatorname{D} \pi(M, N)\left[\dot{X}_{(M, N)}\right]=\dot{X}_{M N^{\mathrm{T}}},
$$

where $\mathrm{D} \pi(X)[\dot{X}]$ denotes the differential of $\pi$ at $X$ applied to $\dot{X}$. This $\dot{X}_{(M, N)}$ is termed the horizontal lift of $\dot{X}_{M N^{\mathrm{T}}}$ at $(M, N)$. (In order to lighten the notation, we use the same symbol for a tangent vector to $\mathcal{M}(p, m \times n)$ and its horizontal lift; the distinction is clear from the subscript or from the context.) If (and only if), for all $(M, N) \in \mathbb{R}_{*}^{m \times p} \times \mathbb{R}_{*}^{n \times p}$, all $\dot{X}_{M N^{\mathrm{T}}}, \check{X}_{M N^{\mathrm{T}}} \in \mathrm{T}_{M N^{\mathrm{T}}} \mathcal{M}(p, m \times n)$, and all $R \in \mathrm{GL}(p)$, it holds that

$$
\bar{g}_{(M, N)}\left(\dot{X}_{(M, N)}, \check{X}_{(M, N)}\right)=\bar{g}_{\left(M R, N R^{-\mathrm{T}}\right)}\left(\dot{X}_{\left(M R, N R^{-\mathrm{T}}\right)}, \check{X}_{\left(M R, N R^{-\mathrm{T}}\right)}\right),
$$

then there is a (unique) Riemannian metric $g$ on $\mathcal{M}(p, m \times n)$ consistently defined by

$$
g_{M N^{\mathrm{T}}}\left(\dot{X}_{M N^{\mathrm{T}}}, \check{X}_{M N^{\mathrm{T}}}\right)=\bar{g}_{(M, N)}\left(\dot{X}_{(M, N)}, \check{X}_{(M, N)}\right) .
$$

The submersion $\pi:\left(\mathbb{R}_{*}^{m \times p} \times \mathbb{R}_{*}^{n \times p}, \bar{g}\right) \rightarrow(\mathcal{M}(p, m \times n), g)$ is then termed a Riemannian submersion, and $(\mathcal{M}(p, m \times n), g)$ is termed a Riemannian quotient manifold of $\left(\mathbb{R}_{*}^{m \times p} \times \mathbb{R}_{*}^{n \times p}, \bar{g}\right)$. (We will sometimes omit the Riemannian metrics in the notation when they are clear from the context or undefined.)

In summary, in order to turn $\pi$ into a Riemannian submersion, we "just" have to choose a Riemannian metric $\bar{g}$ of $\mathbb{R}_{*}^{m \times p} \times \mathbb{R}_{*}^{n \times p}$ that satisfies the invariance condition (10).

\section{$4 \mathcal{M}(p, m \times n)$ as a non-Riemannian quotient manifold}

In this section, we consider on $\mathbb{R}_{*}^{m \times p} \times \mathbb{R}_{*}^{n \times p}$ the Euclidean metric $\bar{g}$, defined by

$$
\bar{g}_{(M, N)}((\dot{M}, \dot{N}),(\check{M}, \check{N})):=\operatorname{trace}\left(\dot{M}^{\mathrm{T}} \check{M}\right)+\operatorname{trace}\left(\dot{N}^{\mathrm{T}} \check{N}\right),
$$

and we show that the invariance condition (10) does not hold. Hence $\pi:\left(\mathbb{R}_{*}^{m \times p} \times \mathbb{R}_{*}^{n \times p}, \bar{g}\right) \rightarrow$ $\mathcal{M}(p, m \times n)$ cannot be turned into a Riemannian submersion.

The horizontal space (8) is

$$
\mathcal{H}_{(M, N)}=\left\{(\dot{M}, \dot{N}): \operatorname{trace}\left(\dot{M}^{\mathrm{T}} M \dot{R}\right)+\operatorname{trace}\left(-\dot{N}^{\mathrm{T}} N \dot{R}^{\mathrm{T}}\right)=0, \forall \dot{R} \in \mathbb{R}^{p \times p}\right\} .
$$


Using the identities trace $(A)=\operatorname{trace}\left(A^{\mathrm{T}}\right)$ and $\operatorname{trace}(A B)=\operatorname{trace}(B A)$, we obtain the iden$\operatorname{tity} \operatorname{trace}\left(\dot{M}^{\mathrm{T}} M \dot{R}\right)+\operatorname{trace}\left(-\dot{N}^{\mathrm{T}} N \dot{R}^{\mathrm{T}}\right)=\operatorname{trace}\left(\left(\dot{R}^{\mathrm{T}}\left(M^{\mathrm{T}} \dot{M}-\dot{N}^{\mathrm{T}} N\right)\right)\right.$. It follows that the following propositions are equivalent:

$$
\begin{gathered}
(\dot{M}, \dot{N}) \in \mathcal{H}_{(M, N)}, \\
M^{\mathrm{T}} \dot{M}=\dot{N}^{\mathrm{T}} N, \\
\exists L_{M}, L_{N}, S:\left\{\begin{array}{l}
\dot{M}=M_{\perp} L_{M}+M\left(M^{\mathrm{T}} M\right)^{-1} S \\
\dot{N}=N_{\perp} L_{N}+N\left(N^{\mathrm{T}} N\right)^{-1} S^{\mathrm{T}},
\end{array}\right.
\end{gathered}
$$

where $M_{\perp}$ denotes an orthonormal $m \times(m-p)$ matrix such that $M^{\mathrm{T}} M_{\perp}=0$, and likewise for $N_{\perp}$.

Let $X=M N^{\mathrm{T}}$ and let $\dot{X}_{M N^{\mathrm{T}}} \in \mathrm{T}_{M N^{\mathrm{T}}} \mathcal{M}(p, m \times n)$. We seek an expression for the horizontal lift $\dot{X}_{(M, N)}=\left(\dot{X}_{\mathrm{M}(M, N)}, \dot{X}_{\mathrm{N}(M, N)}\right)$ of $\dot{X}_{M N^{\mathrm{T}}}$ at $(M, N)$, defined by (9). By a reasoning similar to the one detailed in Section 5.3 below, we obtain

$$
\dot{X}_{\mathrm{M}(M, N)}=\left(\dot{X}_{M N^{\mathrm{T}}} N-M K\right)\left(N^{\mathrm{T}} N\right)^{-1} \quad \text { and } \quad \dot{X}_{\mathrm{N}(M, N)}=\left(\dot{X}_{M N^{\mathrm{T}}}^{\mathrm{T}} M-N K^{\mathrm{T}}\right)\left(M^{\mathrm{T}} M\right)^{-1},
$$

where $K$ solves the Sylvester equation

$$
M^{\mathrm{T}} M K+K N^{\mathrm{T}} N=M^{\mathrm{T}} \dot{X}_{M N^{\mathrm{T}}} N .
$$

One sees by inspection, or by a numerical check, that the invariance condition (10) does not hold, and this concludes the argument.

\section{$5 \mathcal{M}(p, m \times n)$ as a Riemannian quotient manifold of $\mathbb{R}_{*}^{m \times p} \times \mathbb{R}_{*}^{n \times p}$}

In this section, we proceed as in Section 4, but now with a different Riemannian metric $\bar{g}$, defined in (12) below. As we will see, the rationale laid out in Section 4 now leads to the conclusion that $\pi:\left(\mathbb{R}_{*}^{m \times p} \times \mathbb{R}_{*}^{n \times p}, \bar{g}\right) \rightarrow \mathcal{M}(p, m \times n)$, with $\bar{g}$ given by (12) instead of (11), can be turned into a Riemannian submersion. This endows $\mathcal{M}(p, m \times n)$ with a Riemannian metric, $g$. We then work out formulas for the Riemannian gradient and Hessian of $f$ on the Riemannian manifold $(\mathcal{M}(p, m \times n), g)$, and we state the corresponding Newton method.

\subsection{Riemannian metric in total space}

Inspired from the case of the Grassmann manifold viewed as a Riemannian quotient manifold of $\mathbb{R}_{*}^{n \times p}$ [AMS08, Example 3.6.4], we consider the Riemannian metric $\bar{g}$ on $\mathbb{R}_{*}^{m \times p} \times \mathbb{R}_{*}^{n \times p}$ defined by

$$
\bar{g}_{(M, N)}((\dot{M}, \dot{N}),(\check{M}, \check{N})):=\operatorname{trace}\left(\left(M^{\mathrm{T}} M\right)^{-1} \dot{M}^{\mathrm{T}} \check{M}+\left(N^{\mathrm{T}} N\right)^{-1} \dot{N}^{\mathrm{T}} \check{N}\right) .
$$

We now proceed to show that it satisfies the invariance condition (10).

\subsection{Horizontal space}

The elements $(\dot{M}, \dot{N})$ of the horizontal space $\mathcal{H}_{(M, N)}$ (8) are readily found to be characterized by

$$
M^{\mathrm{T}} \dot{M}\left(M^{\mathrm{T}} M\right)^{-1}=\left(N^{\mathrm{T}} N\right)^{-1} \dot{N}^{\mathrm{T}} N .
$$


In other words,

$$
\mathcal{H}_{(M, N)}=\left\{(\dot{M}, \dot{N}) \in \mathbb{R}^{m \times p} \times \mathbb{R}^{n \times p}: N^{\mathrm{T}} N M^{\mathrm{T}} \dot{M}=\dot{N}^{\mathrm{T}} N M^{\mathrm{T}} M\right\} .
$$

\subsection{Horizontal lift}

Let $X=M N^{\mathrm{T}}$ and let $\dot{X}_{M N^{\mathrm{T}}}$ belong to $\mathrm{T}_{M N^{\mathrm{T}}} \mathcal{M}(p, m \times n)$. We seek an expression for the horizontal lift $\dot{X}_{(M, N)}=\left(\dot{X}_{\mathrm{M}(M, N)}, \dot{X}_{\mathrm{N}(M, N)}\right)$ defined in (9). In view of (13), we find that the horizontality condition $\left(\dot{X}_{\mathrm{M}(M, N)}, \dot{X}_{\mathrm{N}(M, N)}\right) \in \mathcal{H}_{(M, N)}$ is equivalent to

$$
\begin{aligned}
\dot{X}_{\mathrm{M}(M, N)} & =M_{\perp} L_{\mathrm{M}}+M\left(M^{\mathrm{T}} M\right)^{-1} K\left(M^{\mathrm{T}} M\right) \\
\dot{X}_{\mathrm{N}(M, N)} & =N_{\perp} L_{\mathrm{N}}+N\left(N^{\mathrm{T}} N\right)^{-1} K^{\mathrm{T}}\left(N^{\mathrm{T}} N\right),
\end{aligned}
$$

where $L_{\mathrm{M}} \in \mathbb{R}^{(m-p) \times p}, L_{\mathrm{N}} \in \mathbb{R}^{(n-p) \times p}$ and $K \in \mathbb{R}^{p \times p}$. Since $\mathrm{D} \pi(M, N)\left[\dot{X}_{\mathrm{M}(M, N)}, \dot{X}_{\mathrm{N}(M, N)}\right] \equiv$ $M \dot{X}_{\mathrm{N}(M, N)}^{\mathrm{T}}+\dot{X}_{\mathrm{M}(M, N)} N^{\mathrm{T}}$, the definition (9) implies that

$$
\dot{X}_{M N^{\mathrm{T}}}=M \dot{X}_{\mathrm{N}(M, N)}^{\mathrm{T}}+\dot{X}_{\mathrm{M}(M, N)} N^{\mathrm{T}} .
$$

Replacing (15) in (16) yields

$$
\dot{X}_{M N^{\mathrm{T}}}=M L_{\mathrm{N}}^{\mathrm{T}} N_{\perp}^{\mathrm{T}}+M\left(N^{\mathrm{T}} N\right) K\left(N^{\mathrm{T}} N\right)^{-1} N^{\mathrm{T}}+M_{\perp} L_{\mathrm{M}} N^{\mathrm{T}}+M\left(M^{\mathrm{T}} M\right)^{-1} K\left(M^{\mathrm{T}} M\right) N^{\mathrm{T}} .
$$

Multiplying (17) on the left by $\left(M^{\mathrm{T}} M\right)^{-1} M^{\mathrm{T}}$ yields

$$
L_{\mathrm{N}}^{\mathrm{T}}=\left(M^{\mathrm{T}} M\right)^{-1} M^{\mathrm{T}} \dot{X}_{M N^{\mathrm{T}}} N_{\perp},
$$

multiplying (17) on the right by $N\left(N^{\mathrm{T}} N\right)^{-1}$ yields

$$
L_{\mathrm{M}}=M_{\perp}^{\mathrm{T}} \dot{X}_{M N^{\mathrm{T}}} N\left(N^{\mathrm{T}} N\right)^{-1},
$$

and multiplying (17) on the left by $M^{\mathrm{T}}$ and on the right by $N$ yields

$$
M^{\mathrm{T}} \dot{X}_{M N^{\mathrm{T}}} N=M^{\mathrm{T}} M N^{\mathrm{T}} N K+K M^{\mathrm{T}} M N^{\mathrm{T}} N .
$$

Replacing (18) into (15) yields

$$
\begin{aligned}
& \dot{X}_{\mathrm{M}(M, N)}=M_{\perp} M_{\perp}^{\mathrm{T}} \dot{X}_{M N^{\mathrm{T}}} N\left(N^{\mathrm{T}} N\right)^{-1}+M\left(M^{\mathrm{T}} M\right)^{-1} K M^{\mathrm{T}} M \\
& \dot{X}_{\mathrm{N}(M, N)}=N_{\perp} N_{\perp}^{\mathrm{T}} \dot{X}_{M N^{\mathrm{T}}}^{\mathrm{T}} M\left(M^{\mathrm{T}} M\right)^{-1}+N\left(N^{\mathrm{T}} N\right)^{-1} K^{\mathrm{T}} N^{\mathrm{T}} N .
\end{aligned}
$$

We can further exploit the identities $M_{\perp} M_{\perp}^{\mathrm{T}}=I-M\left(M^{\mathrm{T}} M\right)^{-1} M^{\mathrm{T}}$, and likewise for $N$, to rewrite (19) as

$$
\begin{gathered}
\dot{X}_{\mathrm{M}(M, N)}=\left(\dot{X}_{M N^{\mathrm{T}}} N-M N^{\mathrm{T}} N K\right)\left(N^{\mathrm{T}} N\right)^{-1} \\
\dot{X}_{\mathrm{N}(M, N)}=\left(\dot{X}_{M N^{\mathrm{T}}}^{\mathrm{T}} M-N M^{\mathrm{T}} M K^{\mathrm{T}}\right)\left(M^{\mathrm{T}} M\right)^{-1} .
\end{gathered}
$$

This result is formalized as follows:

Proposition 5.1 Consider the submersion $\pi$ (4) and the horizontal distribution (14). Let $(M, N) \in \mathbb{R}_{*}^{m \times p} \times \mathbb{R}_{*}^{n \times p}$ and let $\dot{X}_{M N^{\mathrm{T}}}$ be in $\mathrm{T}_{M N^{\mathrm{T}}} \mathcal{M}(p, m \times n)$. Then the horizontal lift of $\dot{X}_{M N^{\mathrm{T}}}$ at $(M, N)$ is $\dot{X}_{(M, N)}=\left(\dot{X}_{\mathrm{M}(M, N)}, \dot{X}_{\mathrm{N}(M, N)}\right)$ given by (20), where $K$ is the solution of the Sylvester equation (18c). 


\subsection{Constitutive equation of horizontal lifts}

A horizontal lift $\dot{X}_{(M, N)}$ fully specifies $\dot{X}_{M N^{\mathrm{T}}}=\mathrm{D} \pi(M, N)\left[\dot{X}_{(M, N)}\right] \in \mathrm{T}_{M N^{\mathrm{T}}} \mathcal{M}(p, m \times n)$ as well as its horizontal lift at any other point of the fiber $\pi^{-1}\left(M N^{\mathrm{T}}\right)$ (6). Let us obtain an expression for $\dot{X}_{\left(M R, N R^{-T}\right)}$ in terms of $\dot{X}_{(M, N)}$. The expression (20) of horizontal lifts yields after routine manipulations

$$
\dot{X}_{\mathrm{M}\left(M R, N R^{-\mathrm{T}}\right)}=\dot{X}_{\mathrm{M}(M, N)} R, \quad \dot{X}_{\mathrm{N}\left(M R, N R^{-\mathrm{T}}\right)}=\dot{X}_{\mathrm{N}(M, N)} R^{-\mathrm{T}} .
$$

We have obtained:

Proposition 5.2 Consider the submersion $\pi$ (44) and the horizontal distribution (14). Then a vector field $\mathbb{R}_{*}^{m \times p} \times \mathbb{R}_{*}^{n \times p} \ni(M, N) \mapsto \dot{X}_{(M, N)} \in \mathbb{R}^{m \times p} \times \mathbb{R}^{n \times p}$ is a horizontal lift if and only if (21) holds for all $(M, N) \in \mathbb{R}_{*}^{m \times p} \times \mathbb{R}_{*}^{n \times p}$ and all $R \in \mathrm{GL}(p)$.

\subsection{Riemannian submersion}

Routine manipulations using (21) yield that $\bar{g}$ (12) satisfies the invariance condition (10). Hence there is a (unique) Riemannian metric $g$ on $\mathcal{M}(p, m \times n)$ that makes

$$
\pi:\left(\mathbb{R}_{*}^{m \times p} \times \mathbb{R}_{*}^{n \times p}, \bar{g}\right) \rightarrow(\mathcal{M}(p, m \times n), g):(M, N) \mapsto M N^{\mathrm{T}}
$$

a Riemannian submersion. The Riemannian metric $g$ is consistently defined by

$$
g_{M N^{\mathrm{T}}}\left(\dot{X}_{M N^{\mathrm{T}}}, \check{X}_{M N^{\mathrm{T}}}\right):=\bar{g}_{(M, N)}\left(\dot{X}_{(M, N)}, \check{X}_{(M, N)}\right) .
$$

\subsection{Horizontal projection}

We will need an expression for the projection $P_{(M, N)}^{\mathrm{h}}(\dot{M}, \dot{N})$ of $(\dot{M}, \dot{N}) \in \mathbb{R}^{m \times p} \times \mathbb{R}^{n \times p}$ onto the horizontal space (14) along the vertical space (7).

Since the projection is along the vertical space, we have

$$
P_{(M, N)}^{\mathrm{h}}(\dot{M}, \dot{N})=\left(\dot{M}+M \dot{R}, \dot{N}-N \dot{R}^{\mathrm{T}}\right)
$$

for some $\dot{R} \in \mathbb{R}^{p \times p}$. It remains to obtain $\dot{R}$ by imposing horizontality of (24). Since horizontal vectors are characterized by (13), we find that (24) is horizontal if and only if

$$
M^{\mathrm{T}}(\dot{M}+M \dot{R})\left(M^{\mathrm{T}} M\right)^{-1}=\left(N^{\mathrm{T}} N\right)^{-1}\left(\dot{N}^{\mathrm{T}}-\dot{R} N^{\mathrm{T}}\right) N
$$

that is,

$$
M^{\mathrm{T}} M \dot{R}\left(M^{\mathrm{T}} M\right)^{-1}+\left(N^{\mathrm{T}} N\right)^{-1} \dot{R} N^{\mathrm{T}} N=-M^{\mathrm{T}} \dot{M}\left(M^{\mathrm{T}} M\right)^{-1}+\left(N^{\mathrm{T}} N\right)^{-1} \dot{N}^{\mathrm{T}} N,
$$

which can be rewritten as the Sylvester equation

$$
N^{\mathrm{T}} N M^{\mathrm{T}} M \dot{R}+\dot{R} N^{\mathrm{T}} N M^{\mathrm{T}} M=-N^{\mathrm{T}} N M^{\mathrm{T}} \dot{M}+\dot{N}^{\mathrm{T}} N M^{\mathrm{T}} M .
$$

In summary:

Proposition 5.3 The projection $P_{(M, N)}^{\mathrm{h}}(\dot{M}, \dot{N})$ of $(\dot{M}, \dot{N}) \in \mathbb{R}^{m \times p} \times \mathbb{R}^{n \times p}$ onto the horizontal space (14) along the vertical space (17) is given by (24) where $\dot{R}$ is the solution of the Sylvester equation (25). 


\subsection{Riemannian connection on the total space}

Since the chosen Riemannian metric $\bar{g}(12)$ on the total space $\mathbb{R}_{*}^{m \times p} \times \mathbb{R}_{*}^{n \times p}$ is not the Euclidean metric (11), it can be expected that the Riemannian connection on $\left(\mathbb{R}_{*}^{m \times p} \times \mathbb{R}_{*}^{n \times p}, \bar{g}\right)$ is not the plain differential. We show that this is indeed the case and we provide a formula for the Riemannian connection $\bar{\nabla}$ on $\left(\mathbb{R}_{*}^{m \times p} \times \mathbb{R}_{*}^{n \times p}, \bar{g}\right)$. The motivation for obtaining this formula is that the Riemannian Newton equation on $(\mathcal{M}(p, m \times n), g)$ requires the Riemannian connection on $(\mathcal{M}(p, m \times n), g)$, which is readily obtained from $\bar{\nabla}$ as we will see in Section 5.8. The general theory of Riemannian connections (also called Levi-Civita connections) can be found in [AMS08, §5.3] or in any Riemannian geometry textbook such as [dC92].

The development relies on Koszul's formula

$$
2 g\left(\nabla_{\chi} \eta, \xi\right)=\partial_{\chi} g(\eta, \xi)+\partial_{\eta} g(\chi, \xi)-\partial_{\xi} g(\chi, \eta)+g([\chi, \eta], \xi)-g([\chi, \xi], \eta)-g([\eta, \xi], \chi) .
$$

After lengthy but routine calculations, we obtain the following expression for the Riemannian connection $\bar{\nabla}$ on $\left(\mathbb{R}_{*}^{m \times p} \times \mathbb{R}_{*}^{n \times p}, \bar{g}\right)$ :

$$
\begin{aligned}
\left(\bar{\nabla}_{\dot{X}} \dot{Y}\right)_{\mathrm{M}}=\partial_{\dot{X}} \dot{Y}_{\mathrm{M}}-\dot{Y}_{\mathrm{M}}\left(M^{\mathrm{T}} M\right)^{-1} \operatorname{sym}\left(\dot{X}_{\mathrm{M}}^{\mathrm{T}} M\right)-\dot{X}_{\mathrm{M}}\left(M^{\mathrm{T}} M\right)^{-1} \operatorname{sym}\left(\dot{Y}_{\mathrm{M}}^{\mathrm{T}} M\right) \\
+M\left(M^{\mathrm{T}} M\right)^{-1} \operatorname{sym}\left(\dot{X}_{\mathrm{M}}^{\mathrm{T}} \dot{Y}_{\mathrm{M}}\right)
\end{aligned}
$$

and

$$
\begin{aligned}
\left(\bar{\nabla}_{\dot{X}} \dot{Y}\right)_{\mathrm{N}}=\partial_{\dot{X}} \dot{Y}_{\mathrm{N}}-\dot{Y}_{\mathrm{N}}\left(N^{\mathrm{T}} N\right)^{-1} \operatorname{sym}\left(\dot{X}_{\mathrm{N}}^{\mathrm{T}} N\right)-\dot{X}_{\mathrm{N}}\left(N^{\mathrm{T}} N\right)^{-1} \operatorname{sym}\left(\dot{Y}_{\mathrm{N}}^{\mathrm{T}} N\right) \\
+N\left(N^{\mathrm{T}} N\right)^{-1} \operatorname{sym}\left(\dot{X}_{\mathrm{N}}^{\mathrm{T}} \dot{Y}_{\mathrm{N}}\right),
\end{aligned}
$$

for all $(M, N) \in \mathbb{R}_{*}^{m \times p} \times \mathbb{R}_{*}^{n \times p}$, all $\dot{X} \in \mathrm{T}_{(M, N)} \mathbb{R}_{*}^{m \times p} \times \mathbb{R}_{*}^{n \times p}$ and all tangent vector fields $\dot{Y}$ on $\mathbb{R}_{*}^{m \times p} \times \mathbb{R}_{*}^{n \times p}$.

\subsection{Connection on the quotient space}

Let $\nabla$ denote the Riemannian connection on the quotient space $\mathcal{M}(p, m \times n)$ endowed with the Riemannian metric $g$ (23). A classical result in the theory of Riemannian submersions (see [’N66, Lemma 1] or [AMS08, §5.3.4]) states that

$$
\left(\nabla_{\dot{X}_{M N T} \mathrm{~T}} \dot{Y}\right)_{(M, N)}=P_{(M, N)}^{\mathrm{h}}\left(\bar{\nabla}_{\dot{X}_{(M, N)}} \dot{Y}\right),
$$

for all $\dot{X}_{M N^{\mathrm{T}}} \in \mathrm{T}_{M N^{\mathrm{T}}} \mathcal{M}(p, m \times n)$ and all tangent tangent vector fields $\dot{Y}$ on $\mathcal{M}(p, m \times n)$. That is, the horizontal lift of the Riemannian connection of the quotient space is given by the horizontal projection (24) of the Riemannian connection (27) of the total space. (The tangent vector field $Y$ on the right-hand side denotes the horizontal lift of the tangent vector field $Y$ of the left-hand side.)

\subsection{Riemannian Newton equation}

For a real-valued function $f$ on a Riemannian manifold $\mathcal{M}$ with Riemannian metric $g$, we let grad $f(x)$ denote the gradient of $f$ at $x \in \mathcal{M}$-defined as the unique tangent vector to $\mathcal{M}$ at 
$x$ that satisfies $g_{x}\left(\operatorname{grad} f(x), \xi_{x}\right)=\mathrm{D} f(x)\left[\xi_{x}\right]$ for all $\xi_{x} \in \mathrm{T}_{x} \mathcal{M}$-and the plain Riemannian Newton equation is given by

$$
\nabla_{\eta_{x}} \operatorname{grad} f=-\operatorname{grad} f(x)
$$

for the unknown $\eta_{x} \in \mathrm{T}_{x} \mathcal{M}$, where $\nabla$ stands for the Riemannian connection; see, e.g., AMS08, $\S 6.2$.

We now turn to the manifold $\mathcal{M}(p, m \times n)$ endowed with the Riemannian metric $g$ (23) and we obtain an expression of the Riemannian Newton equation by means of its horizontal lift through the Riemannian submersion $\pi(22)$. First, on the total space $\mathbb{R}_{*}^{m \times p} \times \mathbb{R}_{*}^{n \times p}$ endowed with the Riemannian metric $\bar{g}(12)$, we readily obtain the following expression for the gradient of $\bar{f}(15)$ :

$$
\operatorname{grad} \bar{f}(M, N)=\left(\partial_{\mathrm{M}} \bar{f}(M, N) M^{\mathrm{T}} M, \partial_{\mathrm{N}} \bar{f}(M, N) N^{\mathrm{T}} N\right),
$$

where $\partial_{\mathrm{M}} \bar{f}(M, N)$ denotes the Euclidean (i.e., classical) gradient of $\bar{f}$ with respect to its first argument, i.e., $\left(\partial_{\mathrm{M}} \bar{f}(M, N)\right)_{i, j}=\left.\frac{\mathrm{d}}{\mathrm{d} t} \bar{f}\left(M+t e_{i} e_{j}^{\mathrm{T}}, N\right)\right|_{t=0}$, and likewise for $\partial_{\mathrm{N}} \bar{f}(M, N)$ with the second argument. Then the horizontal lift of the Newton equation at a point $(M, N)$ of the total space $\mathbb{R}_{*}^{m \times p} \times \mathbb{R}_{*}^{n \times p}$, for the unknown $X_{(M, N)}$ in the horizontal space $\mathcal{H}_{(M, N)}(\underline{14})$, is

$$
P_{(M, N)}^{\mathrm{h}}\left(\bar{\nabla}_{X_{(M, N)}} \operatorname{grad} \bar{f}\right)=-\operatorname{grad} \bar{f}(M, N),
$$

where $P^{\mathrm{h}}$ is the horizontal projection given in Section 5.6 and $\bar{\nabla}$ is the Riemannian connection on $\left(\mathbb{R}_{*}^{m \times p} \times \mathbb{R}_{*}^{n \times p}, \bar{g}\right)$ given in Section 5.7. To obtain (28), we have used the fact (see AMS08, (3.39)]) that $\operatorname{grad} f(M, N)=\operatorname{grad} \bar{f}(M, N)$, where the left-hand side denotes the horizontal lift of $\operatorname{grad} f\left(M N^{\mathrm{T}}\right)$ at $(M, N)$.

Intimidating as it may be in view of the expressions of $P^{\mathrm{h}}$ and $\bar{\nabla}$, the Newton equation (28) is nevertheless merely a linear system of equations. Indeed, $X_{(M, N)} \mapsto P_{(M, N)}^{\mathrm{h}}\left(\bar{\nabla}_{X_{(M, N)}} \operatorname{grad} \bar{f}\right)$ is a linear transformation of the horizontal space $\mathcal{H}_{(M, N)}$. Thus (28) can be solved using "matrix-free" linear solvers such as GMRES. Moreover, in addition to computing the Euclidean gradient of $\bar{f}$ and the Euclidean derivative of the Euclidean gradient of $f$ along $X_{(M, N)}$, computing $P_{(M, N)}^{\mathrm{h}}\left(\bar{\nabla}_{X_{(M, N)}} \operatorname{grad} \bar{f}\right)$ requires only $O\left(p^{2}(m+n+p)\right)$ flops.

\subsection{Newton's method}

In order to spell out on $(\mathcal{M}(p, m \times n), g)$ the Riemannian Newton method as defined in AMS08 $\S 6.2]$, the last missing ingredient is a retraction $R$ that turns the Newton vector $\dot{X}_{M N^{\mathrm{T}}}$ into an updated iterate $R_{M N^{\mathrm{T}}} \dot{X}_{M N^{\mathrm{T}}}$ in $\mathcal{M}(p, m \times n)$. The general definition of a retraction can be found in [AMS08, §4.1].

The quintessential retraction on a Riemannian manifold is the Riemannian exponential; see [AMS08, §5.4]. However, computing the Riemannian exponential amounts to solving the differential equation $\nabla_{\dot{X}} \dot{X}=0$, which may not admit a closed-form solution. In the case of $(\mathcal{M}(p, m \times n), g)$, we are not aware of such a closed-form solution, and this makes the exponential retraction impractical.

Fortunately, other retractions are readily available. A retraction on $\mathcal{M}(p, m \times n)$ is given by

$$
R_{M N^{\mathrm{T}}}\left(\dot{X}_{M N^{\mathrm{T}}}\right):=\left(M+\dot{X}_{\mathrm{M}(M, N)}\right)\left(N+\dot{X}_{\mathrm{N}(M, N)}\right)^{\mathrm{T}},
$$

where $\dot{X}_{\mathrm{M}(M, N)}$ and $\dot{X}_{\mathrm{N}(M, N)}$ are horizontal lifts as defined in Proposition [5.1] It is readily checked that the definition is consistent, i.e., it depends on $M N^{\mathrm{T}}$ and not on the specific choices of $(M, N)$ in the fiber (6). 
With all these elements in place, we can describe Newton's method as follows.

Theorem 5.4 (Riemannian Newton on $\mathcal{M}(p, m \times n)$ with Riemannian metric (23)) Let $f$ be a real-valued function on the Riemannian manifold $\mathcal{M}(p, m \times n)$ (1), endowed with the Riemannian metric $g$ (23), with the associated Riemannian connection, and with the retraction (29). Then the Riemannian Newton method for $f$ maps $M N^{\mathrm{T}} \in \mathcal{M}(p, m \times n)$ to $\left(M+\dot{X}_{\mathrm{M}}\right)\left(N+\dot{X}_{\mathrm{N}}\right)^{\mathrm{T}}$, where $\left(\dot{X}_{\mathrm{M}}, \dot{X}_{\mathrm{N}}\right)$ is the solution $\dot{X}_{(M, N)}$ of the Newton equation (28).

Note that, in practice, it is not necessary to form $M N^{\mathrm{T}}$. Given an initial point $M_{0} N_{0}^{\mathrm{T}}$, one can instead generate a sequence $\left\{\left(M_{k}, N_{k}\right)\right\}$ in $\mathbb{R}_{*}^{m \times p} \times \mathbb{R}_{*}^{n \times p}$ by applying the iteration $\operatorname{map}(M, N) \mapsto\left(M+\dot{X}_{\mathrm{M}}, N+\dot{X}_{\mathrm{N}}\right)$. The Newton sequence on $\mathcal{M}(p, m \times n)$ is then $\left\{M_{k} N_{k}^{\mathrm{T}}\right\}$, and it depends on $M_{0} N_{0}^{\mathrm{T}}$ but not on the particular $M_{0}$ and $N_{0}$.

The following convergence result follows directly from the general convergence analysis of the Riemannian Newton method AMS08, Theorem 6.3.2]. A critical point of $f$ : $\mathcal{M}(p, m \times n) \rightarrow \mathbb{R}$ is a point $X_{*}$ where grad $f\left(X_{*}\right)=0$. It is termed nondegenerate if the Hes$\operatorname{sian} \mathrm{T}_{X_{*}} \mathcal{M}(p, m \times n) \ni \dot{X} \mapsto \nabla_{\dot{X}} \operatorname{grad} f \in \mathrm{T}_{X_{*}} \mathcal{M}(p, m \times n)$ is invertible. These definitions do not depend on the Riemannian metric nor on the affine connection $\nabla$.

Theorem 5.5 (quadratic convergence) Let $X_{*}$ be a nondegenerate critical point of $f$. Then there exists a neighborhood $\mathcal{U}$ of $X_{*}$ in $\mathcal{M}(p, m \times n)$ such that, for all initial iterate $X_{0} \in \mathcal{U}$, the iteration described in Theorem 5.4 generates an infinite sequence $\left\{X_{k}\right\}$ converging superlinearly (at least quadratically) to $X_{*}$.

\section{$6 \mathcal{M}(p, m \times n)$ as a Riemannian quotient manifold with an or- thonormal factor}

We now follow the second plan of action mentioned at the end of Section 1 . Bear in mind that the meaning of much of the notation introduced above will be superseded by new definitions below.

\subsection{A smaller total space}

Let

$$
\operatorname{St}(p, m)=\left\{M \in \mathbb{R}^{m \times p}: M^{\mathrm{T}} M=I_{p}\right\},
$$

denote the Stiefel manifold of orthonormal $m \times p$ matrices. For all $X \in \mathcal{M}(p, m \times n)$, there exists $(M, N)$ with $M$ orthonormal such that $X=M N^{\mathrm{T}}$. To see this, take $(M, N) \in$ $\mathbb{R}_{*}^{m \times p} \times \mathbb{R}_{*}^{n \times p}$ such that $X=M N^{\mathrm{T}}$, let $M=Q R$ be a $\mathrm{QR}$ decomposition of $M$, where $R$ is invertible since $M$ has full rank, and observe that $X=M R^{-1}\left(N R^{\mathrm{T}}\right)^{\mathrm{T}}=Q\left(N R^{\mathrm{T}}\right)^{\mathrm{T}}$. Hence

$$
\pi: \operatorname{St}(p, m) \times \mathbb{R}_{*}^{n \times p} \rightarrow \mathcal{M}(p, m \times n):(M, N) \mapsto M N^{\mathrm{T}}
$$

is a smooth surjective map between two manifolds.

As in Section 2, but now with the restricted total space $\operatorname{St}(p, m) \times \mathbb{R}_{*}^{n \times p}$, we show that $\pi$ (31) is a submersion. The tangent space at $M$ to $\operatorname{St}(p, m)$ is given by (see AMS08, Example 3.5.2])

$$
\begin{aligned}
\mathrm{T}_{M} \operatorname{St}(p, m) & =\left\{\dot{M} \in \mathbb{R}^{m \times p}: M^{\mathrm{T}} \dot{M}+\dot{M}^{\mathrm{T}} M=0\right\} \\
& =\left\{M \Omega+M_{\perp} W: \Omega=-\Omega^{\mathrm{T}} \in \mathbb{R}^{p \times p}, W \in \mathbb{R}^{(m-p) \times p}\right\},
\end{aligned}
$$


and we have

$$
\mathrm{T}_{(M, N)} \operatorname{St}(p, m) \times \mathbb{R}_{*}^{n \times p}=\left(\mathrm{T}_{M} \operatorname{St}(p, m)\right) \times \mathbb{R}^{n \times p} .
$$

For all $(M, N) \in \operatorname{St}(p, m) \times \mathbb{R}_{*}^{n \times p}$ and all $(\dot{M}, \dot{N}) \in \mathrm{T}_{(M, N)} \operatorname{St}(p, m) \times \mathbb{R}_{*}^{n \times p}$, we have that $\mathrm{D} \pi(M, N)[(\dot{M}, \dot{N})]=\dot{M} N^{\mathrm{T}}+M \dot{N}^{\mathrm{T}}$. Here again, we can work in a coordinate system where $M=\left[\begin{array}{ll}I & 0\end{array}\right]^{\mathrm{T}}$ and $N=\left[\begin{array}{ll}I & 0\end{array}\right]^{\mathrm{T}}$. We have that $\{\mathrm{D} \pi(M, N)[(\dot{M}, \dot{N})]:(\dot{M}, \dot{N}) \in$ $\left.\mathrm{T}_{(M, N)} \operatorname{St}(p, m) \times \mathbb{R}_{*}^{n \times p}\right\}=\left\{\left[\begin{array}{cc}\Omega+N_{1}^{\mathrm{T}} & N_{2}^{\mathrm{T}} \\ W & 0\end{array}\right]: \Omega=-\Omega^{\mathrm{T}} \in \mathbb{R}^{p \times p}, N_{1} \in \mathbb{R}^{p \times p}, N_{2} \in \mathbb{R}^{(n-p) \times p}, W \in\right.$ $\left.\mathbb{R}^{(m-p) \times p}\right\}$, a linear subspace of dimension $p^{2}+(n-p) p+(m-p) p=p(m+n-p)$, which is the dimension of $\mathcal{M}(p, m \times n)$. Hence $\pi$ (31) is a submersion.

The fiber of $\pi$ (31) at $M N^{\mathrm{T}}$ is now

$$
\pi^{-1}\left(M N^{\mathrm{T}}\right)=\{(M R, N R): R \in \mathrm{O}(p)\},
$$

where

$$
\mathrm{O}(p)=\left\{R \in \mathbb{R}^{p \times p}: R^{\mathrm{T}} R=I_{p}\right\}
$$

denotes the orthogonal group of degree $p$.

The vertical space $\mathcal{V}_{(M, N)}$ at a point $(M, N) \in \mathbb{R}_{*}^{m \times p} \times \mathbb{R}_{*}^{n \times p}$, i.e., the tangent space to the fiber $\pi^{-1}\left(M N^{\mathrm{T}}\right)$ at $(M, N)$, is given by

$$
\mathcal{V}_{(M, N)}=\left\{(M \Omega, N \Omega): \Omega=-\Omega^{\mathrm{T}} \in \mathbb{R}^{p \times p}\right\} .
$$

\subsection{Riemannian metric in total space}

We consider $\operatorname{St}(p, m) \times \mathbb{R}_{*}^{n \times p}$ as a Riemannian submanifold of the Euclidean space $\mathbb{R}^{m \times p} \times \mathbb{R}^{n \times p}$. This endows $\operatorname{St}(p, m) \times \mathbb{R}_{*}^{n \times p}$ with the Riemannian metric $\bar{g}$ defined by

$$
\bar{g}_{(M, N)}((\dot{M}, \dot{N}),(\check{M}, \check{N})):=\operatorname{trace}\left(\dot{M}^{\mathrm{T}} \check{M}+\dot{N}^{\mathrm{T}} \check{N}\right)
$$

for all $(\dot{M}, \dot{N})$ and $(\check{M}, \check{N})$ in $\mathrm{T}_{(M, N)} \operatorname{St}(p, m) \times \mathbb{R}_{*}^{n \times p}$.

Adapting the rationale of Section 5, we will obtain in Section 6.6 below that, with this $\bar{g}$, $\pi$ (31) can be turned into a Riemannian submersion.

\subsection{Horizontal space}

The horizontal space $\mathcal{H}_{(M, N)}$ is the orthogonal complement to $\mathcal{V}_{(M, N)}(33)$ in $\mathrm{T}_{(M, N)} \operatorname{St}(p, m) \times \mathbb{R}_{*}^{n \times p}$ with respect to $\bar{g}$ (34). The following propositions are equivalent:

$$
\begin{gathered}
(\dot{M}, \dot{N}) \in \mathcal{H}_{(M, N)}, \\
\dot{M} \in \mathrm{T}_{M} \operatorname{St}(p, n), \dot{N} \in \mathbb{R}^{n \times p}, \operatorname{tr}\left(\tilde{\Omega}^{\mathrm{T}}\left(M^{\mathrm{T}} \dot{M}+N^{\mathrm{T}} \dot{N}\right)\right)=0, \forall \tilde{\Omega}=-\tilde{\Omega}^{\mathrm{T}}, \\
M^{\mathrm{T}} \dot{M}=-\left(M^{\mathrm{T}} \dot{M}\right)^{\mathrm{T}}, M^{\mathrm{T}} \dot{M}+N^{\mathrm{T}} \dot{N}=\left(M^{\mathrm{T}} \dot{M}+N^{\mathrm{T}} \dot{N}\right)^{\mathrm{T}}, \\
\dot{M}=M \Omega+M_{\perp} W, \dot{N}=N\left(N^{\mathrm{T}} N\right)^{-1}(-\Omega+S)+N_{\perp} L,
\end{gathered}
$$

with $W \in \mathbb{R}^{(m-p) \times p}, \Omega=-\Omega^{\mathrm{T}} \in \mathbb{R}^{p \times p}, S=S^{\mathrm{T}} \in \mathbb{R}^{p \times p}, L \in \mathbb{R}^{(n-p) \times p}$. In summary,

$$
\mathcal{H}_{(M, N)}=\left\{(\dot{M}, \dot{N}): M^{\mathrm{T}} \dot{M}=-\left(M^{\mathrm{T}} \dot{M}\right)^{\mathrm{T}}, M^{\mathrm{T}} \dot{M}+N^{\mathrm{T}} \dot{N}=\left(M^{\mathrm{T}} \dot{M}+N^{\mathrm{T}} \dot{N}\right)^{\mathrm{T}}\right\} .
$$




\subsection{Horizontal lift}

Proceeding as in Section 5.3 but now with the horizontal space (36) and taking into account that $M^{\mathrm{T}} M=I$, we obtain that the horizontal lift of $\dot{X}_{M N^{\mathrm{T}}} \in \mathrm{T}_{M N^{\mathrm{T}}} \operatorname{St}(p, m) \times \mathbb{R}_{*}^{n \times p}$ is given by

$$
\begin{gathered}
\dot{X}_{\mathrm{M}(M, N)}=M \Omega+M_{\perp} M_{\perp}^{\mathrm{T}} \dot{X}_{M N^{\mathrm{T}}} N\left(N^{\mathrm{T}} N\right)^{-1} \\
\dot{X}_{\mathrm{N}(M, N)}=N\left(N^{\mathrm{T}} N\right)^{-1}(S-\Omega)+N_{\perp} N_{\perp}^{\mathrm{T}} \dot{X}_{M N^{\mathrm{T}}}^{\mathrm{T}} M \\
\text { where } \quad \Omega\left(N^{\mathrm{T}} N+I\right)+S=M^{\mathrm{T}} \dot{X}_{M N^{\mathrm{T}}} N, \Omega=-\Omega^{\mathrm{T}}, S=S^{\mathrm{T}} .
\end{gathered}
$$

Equation (37c) is equivalent to

$$
\begin{gathered}
\Omega\left(N^{\mathrm{T}} N+I\right)+\left(N^{\mathrm{T}} N+I\right) \Omega=M^{\mathrm{T}} \dot{X}_{M N^{\mathrm{T}}} N-N^{\mathrm{T}} \dot{X}_{M N^{\mathrm{T}}}^{\mathrm{T}} M, \\
S=M \dot{X}_{M N^{\mathrm{T}}} N-\Omega\left(N^{\mathrm{T}} N+I\right) .
\end{gathered}
$$

As for the first two equations of (37), using (37c), they can be rewritten as

$$
\begin{gathered}
\dot{X}_{\mathrm{M}(M, N)}=\dot{X}_{M N^{\mathrm{T}}} N\left(N^{\mathrm{T}} N\right)^{-1}-M(\Omega+S)\left(N^{\mathrm{T}} N\right)^{-1} \\
\dot{X}_{\mathrm{N}(M, N)}=\dot{X}_{M N^{\mathrm{T}}}^{\mathrm{T}} M+N \Omega .
\end{gathered}
$$

In summary,

Proposition 6.1 Consider the submersion $\pi$ (31) and the horizontal distribution (36). Let $(M, N) \in \operatorname{St}(p, m) \times \mathbb{R}_{*}^{n \times p}$ and let $\dot{X}_{M N^{\mathrm{T}}} \in \mathrm{T}_{M N^{\mathrm{T}}} \mathcal{M}(p, m \times n)$. Then the horizontal lift of $\dot{X}_{M N^{\mathrm{T}}}$ at $(M, N)$ is $\dot{X}_{(M, N)}=\left(\dot{X}_{\mathrm{M}(M, N)}, \dot{X}_{\mathrm{N}(M, N)}\right)$ given by (39), where $\Omega$ is the solution of the Sylvester equation (38a) and $S$ is given by (38b).

\subsection{Constitutive equation of horizontal lifts}

From Proposition 6.1, routine manipulations lead to the following constitutive equation for horizontal lifts:

$$
\dot{X}_{\mathrm{M}(M R, N R)}=\dot{X}_{\mathrm{M}(M, N)} R, \quad \dot{X}_{\mathrm{N}(M R, N R)}=\dot{X}_{\mathrm{N}(M, N)} R .
$$

Hence we have the following counterpart of Proposition 5.2.

Proposition 6.2 Consider the submersion $\pi$ (31) and the horizontal distribution (36). Then a tangent vector field $\operatorname{St}(p, m) \times \mathbb{R}_{*}^{n \times p} \ni(M, N) \mapsto \dot{X}_{(M, N)} \in \mathrm{T}_{(M, N)} \operatorname{St}(p, m) \times \mathbb{R}_{*}^{n \times p}$ is a horizontal lift if and only if (40) holds for all $(M, N) \in \operatorname{St}(p, m) \times \mathbb{R}_{*}^{n \times p}$ and all $R \in \mathrm{O}(p)$.

\subsection{Riemannian submersion}

From Proposition 6.2 and the properties of the trace, it is direct that $\bar{g}$ (34) satisfies the invariance condition

$$
\bar{g}_{(M, N)}\left(\dot{X}_{(M, N)}, \check{X}_{(M, N)}\right)=\bar{g}_{(M R, N R)}\left(\dot{X}_{(M R, N R)}, \check{X}_{(M R, N R)}\right) .
$$

Hence one consistently defines a Riemannian metric $g$ on $\mathcal{M}(p, m \times n)$ by

$$
g_{M N^{\mathrm{T}}}\left(\dot{X}_{M N^{\mathrm{T}}}, \check{X}_{M N^{\mathrm{T}}}\right)=\bar{g}_{(M, N)}\left(\dot{X}_{(M, N)}, \check{X}_{(M, N)}\right),
$$

and $\pi:\left(\operatorname{St}(p, m) \times \mathbb{R}_{*}^{n \times p}, \bar{g}\right) \rightarrow(\mathcal{M}(p, m \times n), g)$ is a Riemannian submersion. 


\subsection{Horizontal projection}

We now obtain an expression for the projection $P_{(M, N)}^{\mathrm{h}}(\dot{M}, \dot{N})$ of $(\dot{M}, \dot{N}) \in \mathrm{T}_{(M, N)} \operatorname{St}(p, m) \times \mathbb{R}_{*}^{n \times p}$ onto the horizontal space (36) along the vertical space (33). Since the projection is along the vertical space, we have

$$
P_{(M, N)}^{\mathrm{h}}(\dot{M}, \dot{N})=(\dot{M}+M \Omega, \dot{N}+N \Omega)
$$

for some $\Omega=-\Omega^{\mathrm{T}} \in \mathbb{R}^{p \times p}$. It remains to obtain $\Omega$ by imposing horizontality of (43). The characterization of horizontal vectors given in (35) yields the Sylvester equation

$$
\left(N^{\mathrm{T}} N+I\right) \Omega+\Omega\left(N^{\mathrm{T}} N+I\right)=\dot{M}^{\mathrm{T}} M-M^{\mathrm{T}} \dot{M}+\dot{N}^{\mathrm{T}} N-N^{\mathrm{T}} \dot{N} .
$$

In summary:

Proposition 6.3 The projection $P_{(M, N)}^{\mathrm{h}}(\dot{M}, \dot{N})$ of $(\dot{M}, \dot{N}) \in \mathrm{T}_{(M, N)} \mathrm{St}(p, m) \times \mathbb{R}_{*}^{n \times p}$ onto the horizontal space (36) along the vertical space (33) is given by (43) where $\Omega$ is the solution of the Sylvester equation (44).

\subsection{Riemannian connection on the total space}

Let $P_{M}^{\mathrm{St}}$ denote the orthogonal projection from $\mathbb{R}^{m \times p}$ onto $\mathrm{T}_{M} \mathrm{St}(p, n)$, given by (see $\underline{\mathrm{AMS} 08}$, Example 5.3.2])

$$
P_{M}^{\mathrm{St}} \dot{M}=\left(I-M M^{\mathrm{T}}\right) \dot{M}+M \operatorname{skew}\left(M^{\mathrm{T}} \dot{M}\right)=\dot{M}-M \operatorname{sym}\left(M^{\mathrm{T}} \dot{M}\right),
$$

where $\operatorname{skew}(Z):=\frac{1}{2}\left(Z-Z^{\mathrm{T}}\right)$ and $\operatorname{sym}(Z):=\frac{1}{2}\left(Z+Z^{\mathrm{T}}\right)$. We also let $P_{(M, N)}^{\mathrm{St} \times \mathbb{R}}$ denote the orthogonal projection from $\mathbb{R}^{m \times p} \times \mathbb{R}^{n \times p}$ onto $\mathrm{T}_{(M, N)} \mathrm{St}(p, m) \times \mathbb{R}_{*}^{n \times p}$, given by

$$
P_{(M, N)}^{\mathrm{St} \times \mathbb{R}}(\dot{M}, \dot{N})=\left(P_{M}^{\mathrm{St}} \dot{M}, \dot{N}\right) .
$$

Since $\operatorname{St}(p, m) \times \mathbb{R}_{*}^{n \times p}$, endowed with the Riemannian metric $\bar{g}$ (34), is a Riemannian submanifold of the Euclidean space $\mathbb{R}^{m \times p} \times \mathbb{R}^{n \times p}$, a classical result of Riemannian geometry (see [AMS08, §5.3.3]) yields that the Riemannian connection $\bar{\nabla}$ on $\left(\operatorname{St}(p, m) \times \mathbb{R}_{*}^{n \times p}, \bar{g}\right)$ is given by

$$
\bar{\nabla}_{\dot{X}} \dot{Y}=P_{(M, N)}^{\mathrm{St} \times \mathbb{R}} \partial_{\dot{X}} \dot{Y}
$$

that is,

$$
\begin{aligned}
& \left(\bar{\nabla}_{\dot{X}} \dot{Y}\right)_{\mathrm{M}}=P_{M}^{\mathrm{St}}\left(\partial_{\dot{X}} \dot{Y}_{\mathrm{M}}\right) \\
& \left(\bar{\nabla}_{\dot{X}} \dot{Y}\right)_{\mathrm{N}}=\partial_{\dot{X}} \dot{Y}_{\mathrm{N}}
\end{aligned}
$$

for all $(M, N) \in \operatorname{St}(p, m) \times \mathbb{R}_{*}^{n \times p}$, all $\dot{X} \in \mathrm{T}_{(M, N)} \operatorname{St}(p, m) \times \mathbb{R}_{*}^{n \times p}$ and all vector fields $\dot{Y}$ on $\operatorname{St}(p, m) \times \mathbb{R}_{*}^{n \times p}$. 


\subsection{Connection on the quotient space}

As in Section 5.8, we can now provide an expression for the Riemannian connection $\nabla$ on the manifold $\mathcal{M}(p, m \times n)$ endowed with the Riemannian metric $g$ (42):

$$
\begin{aligned}
\left(\nabla_{\dot{X}_{M N \mathrm{~T}}} \dot{Y}\right)_{(M, N)} & =P_{(M, N)}^{\mathrm{h}}\left(\bar{\nabla}_{\dot{X}_{(M, N)}} \dot{Y}\right) \\
& =P_{(M, N)}^{\mathrm{h}} P_{(M, N)}^{\mathrm{St} \times \mathbb{R}} \partial_{\dot{X}} \dot{Y},
\end{aligned}
$$

with $P^{\mathrm{h}}$ as in (43) and $P^{\mathrm{St} \times \mathbb{R}}$ as in (46). (Observe that $\dot{Y}$ of the right-hand side is the horizontal lift of $\dot{Y}$ of the left-hand side.)

\subsection{Riemannian Newton equation}

Given $f: \mathcal{M}(p, m \times n) \rightarrow \mathbb{R}$, define $\bar{f}=f \circ \pi$, i.e.,

$$
\bar{f}: \operatorname{St}(p, m) \times \mathbb{R}_{*}^{n \times p} \rightarrow \mathbb{R}:(M, N) \mapsto f\left(M N^{\mathrm{T}}\right),
$$

and define

$$
\overline{\bar{f}}: \mathbb{R}_{*}^{m \times p} \times \mathbb{R}_{*}^{n \times p} \rightarrow \mathbb{R}:(M, N) \mapsto f\left(M N^{\mathrm{T}}\right) .
$$

Let $\operatorname{grad} \overline{\bar{f}}$ denote the Euclidean gradient of $\overline{\bar{f}}$. We have (see [AMS08, (3.37)])

$$
\operatorname{grad} \bar{f}(M, N)=P_{(M, N)}^{\mathrm{St} \times \mathbb{R}} \operatorname{grad} \overline{\bar{f}}(M, N)
$$

and (see [AMS08, (3.39)])

$$
\operatorname{grad} f(M, N)=\operatorname{grad} \bar{f}(M, N),
$$

where the left-hand side stands for the horizontal lift at $(M, N)$ of $\operatorname{grad} f\left(M N^{\mathrm{T}}\right)$.

We can now obtain the counterpart of the (lifted) Newton equation (28) with normalization on the M factor:

$$
P_{(M, N)}^{\mathrm{h}}\left(\bar{\nabla}_{X_{(M, N)}} \operatorname{grad} \bar{f}\right)=-\operatorname{grad} \bar{f}(M, N),
$$

where $P^{\mathrm{h}}$ is the horizontal projection given in Section 6.7, $\bar{\nabla}$ is the Riemannian connection on $\left(\mathbb{R}_{*}^{m \times p} \times \mathbb{R}_{*}^{n \times p}, \bar{g}\right)$ given in Section 6.8, and grad $\bar{f}$ is obtained from the Euclidean gradient of $\bar{f}$ from (48).

The Newton equation (49) can be considered less intricate than in the non-orthonormal case (28) because the expression for $\bar{\nabla}$ in (47) is simpler than in (27). In any case, the discussion that follows (28) applies equally: the Newton equation is merely a linear system of equations, and the Riemannian overhead requires only $O\left(p^{2}(m+n+p)\right)$ flops.

\subsection{Newton's method}

Another reward that comes with the orthonormalization of the M factor is that the Riemannian exponential with respect to $g$ (42) admits a closed-form expression. First, we point out that, in view of [EAS98, $\S 2.2 .2]$, the Riemannian exponential on $\operatorname{St}(p, m) \times \mathbb{R}_{*}^{n \times p}$ for the Riemannian metric $\bar{g}(34)$ is given by

$$
\operatorname{Exp}_{(M, N)}(\dot{M}, \dot{N})=\left(\left[\begin{array}{cc}
M & \dot{M}
\end{array}\right] \exp \left[\begin{array}{cc}
A & -S \\
I & A
\end{array}\right] I_{2 p, p} \exp (-A), N+\dot{N}\right),
$$


where $A:=M^{\mathrm{T}} \dot{M}$ and $S:=\dot{M}^{\mathrm{T}} \dot{M}$, and where exp stands for the matrix exponential (expm in Matlab). Second, since by [’N83, Corollary 7.46] horizontal geodesics in $\left(\operatorname{St}(p, m) \times \mathbb{R}_{*}^{n \times p}, \bar{g}\right)$ map to geodesics in $(\mathcal{M}(p, m \times n), g)$, we have that

$$
\operatorname{Exp}_{M N^{\mathrm{T}}}\left(\dot{X}_{M N^{\mathrm{T}}}\right)=\pi\left(\operatorname{Exp}_{(M, N)}\left(\dot{X}_{\mathrm{M}(M, N)}, \dot{X}_{\mathrm{N}(M, N)}\right)\right),
$$

with $\left(\dot{X}_{\mathrm{M}(M, N)}, \dot{X}_{\mathrm{N}(M, N)}\right)$ as in Proposition 6.1] (In (51), Exp on the right-hand side is given by (50) and Exp on the left-hand side denotes the Riemannian exponential of $(\mathcal{M}(p, m \times n), g)$.)

Observe that the matrix exponential is applied in (50) to matrices of size $2 p \times 2 p$ and $p \times p$; hence, when $p \ll m$, the cost of computing the $\mathrm{M}$ component of (50) is comparable to the cost of computing the simple sum $M+\dot{M}$. Note also that, in practice, the M component of the Newton iterates may gradually depart from orthonormality due to the accumulation of numerical errors; a remedy is to restore orthonormality by taking the $\mathrm{Q}$ factor of the unique $\mathrm{QR}$ decomposition where the diagonal of the $\mathrm{R}$ factor is positive.

We can now formally describe Newton's method in the context of this Section 6 .

Theorem 6.4 (Riemannian Newton on $\mathcal{M}(p, m \times n)$ with Riemannian metric (42)) Let $f$ be a real-valued function on the Riemannian manifold $\mathcal{M}(p, m \times n)$ (1), endowed with the Riemannian metric $g$ (42), with the associated Riemannian connection, and with the exponential retraction (51). Then the Riemannian Newton method for $f$ maps $M N^{\mathrm{T}} \in$ $\mathcal{M}(p, m \times n)$ to $\pi\left(\operatorname{Exp}_{(M, N)}\left(\dot{X}_{\mathrm{M}}, \dot{X}_{\mathrm{N}}\right)\right)$, where $\pi$ is given in (31), Exp is defined in (50), and $\left(\dot{X}_{\mathrm{M}}, \dot{X}_{\mathrm{N}}\right)$ is the solution $\dot{X}_{(M, N)}$ of the Newton equation (49).

The quadratic convergence result in Theorem 5.5 still holds, replacing the reference to Theorem 5.4 by a reference to Theorem 6.4

\section{Conclusion}

We have reached the end of a technical hike that led us to give in Theorem 6.4 what is, to the best of our knowledge, the first closed-form description of a purely Riemannian Newton method on the set of all matrices of fixed dimension and rank. By "closed-form", we mean that, besides calling an oracle for Euclidean first and second derivatives, the method only needs to perform elementary matrix operations, solve linear systems of equations, and compute (small-size) matrix exponentials. By "purely Riemannian", we mean that it uses the tools provided by Riemannian geometry, namely, the Riemannian connection (instead of any other affine connection) and the Riemannian exponential (instead of any other retraction).

The developments strongly rely on the theory of Riemannian submersions and are based on factorizations of low rank matrices $X$ as $M N^{\mathrm{T}}$, where one of the factors is orthonormal. Relaxing the orthonormality constraint is more appealing for its symmetry (the two factors are treated alike), but it did not allow us to obtain a closed-form expression for the Riemannian exponential.

\section{Acknowledgement}

The authors are grateful to Nicolas Boumal for several helpful comments on a preliminary version of this work. 


\section{References}

$\left[\mathrm{ADM}^{+} 02\right]$ Roy L. Adler, Jean-Pierre Dedieu, Joseph Y. Margulies, Marco Martens, and Mike Shub. Newton's method on Riemannian manifolds and a geometric model for the human spine. IMA J. Numer. Anal., 22(3):359-390, July 2002.

[ADY09] Luca Amodei, Jean-Pierre Dedieu, and Jean-Claude Yakoubsohn. A dynamical approach to lowrank approximation of a matrix, 2009. communication at the 14th Belgian-French-German Conference on Optimization, 14-18 September 2009.

[AMR88] R. Abraham, J. E. Marsden, and T. Ratiu. Manifolds, Tensor Analysis, and Applications, volume 75 of Applied Mathematical Sciences. Springer-Verlag, New York, second edition, 1988.

[AMS08] P.-A. Absil, R. Mahony, and R. Sepulchre. Optimization Algorithms on Matrix Manifolds. Princeton University Press, Princeton, NJ, 2008.

[BA11] N. Boumal and P.-A. Absil. RTRMC: A Riemannian trust-region method for low-rank matrix completion. In J. Shawe-Taylor, R.S. Zemel, P. Bartlett, F.C.N. Pereira, and K.Q. Weinberger, editors, Advances in Neural Information Processing Systems 24 (NIPS), pages 406-414. 2011.

[dC92] Manfredo Perdigão do Carmo. Riemannian geometry. Mathematics: Theory \& Applications. Birkhäuser Boston Inc., Boston, MA, 1992. Translated from the second Portuguese edition by Francis Flaherty.

[DKM12] Wei Dai, E. Kerman, and O. Milenkovic. A geometric approach to low-rank matrix completion. Information Theory, IEEE Transactions on, 58(1):237 -247, jan. 2012.

[DMK11] Wei Dai, O. Milenkovic, and E. Kerman. Subspace evolution and transfer (set) for low-rank matrix completion. Signal Processing, IEEE Transactions on, 59(7):3120 -3132, july 2011.

[DS83] John E. Dennis, Jr. and Robert B. Schnabel. Numerical methods for unconstrained optimization and nonlinear equations. Prentice Hall Series in Computational Mathematics. Prentice Hall Inc., Englewood Cliffs, NJ, 1983.

[EAS98] Alan Edelman, Tomás A. Arias, and Steven T. Smith. The geometry of algorithms with orthogonality constraints. SIAM J. Matrix Anal. Appl., 20(2):303-353, 1998.

[GR85] A. Griewank and G. W. Reddien. The approximation of simple singularities. In Numerical boundary value ODEs (Vancouver, B.C., 1984), volume 5 of Progr. Sci. Comput., pages 245-259. Birkhäuser Boston, Boston, MA, 1985.

[HM94] Uwe Helmke and John B. Moore. Optimization and Dynamical Systems. Communications and Control Engineering Series. Springer-Verlag London Ltd., London, 1994. With a foreword by R. Brockett.

[HS95] Uwe Helmke and Mark A. Shayman. Critical points of matrix least squares distance functions. Linear Algebra and its Applications, 215(0):1 - 19, 1995.

[KMO10] Raghunandan H. Keshavan, Andrea Montanari, and Sewoong Oh. Matrix completion from noisy entries, 2010. arXiv:0906.2027v2.

[Mey11] G. Meyer. Geometric optimization algorithms for linear regression on fixed-rank matrices. PhD thesis, University of Liège, 2011.

[MMBS11] B. Mishra, G. Meyer, F. Bach, and R. Sepulchre. Low-rank optimization with trace norm penalty, 2011. arXiv:1112.2318v1.

[MMS11] B. Mishra, G. Meyer, and R. Sepulchre. Low-rank optimization for distance matrix completion. In Decision and Control and European Control Conference (CDC-ECC), 2011 50th IEEE Conference on, pages $4455-4460$, dec. 2011.

[O'N66] Barrett O'Neill. The fundamental equations of a submersion. Michigan Math. J., 13:459-469, 1966.

[O'N83] Barrett O'Neill. Semi-Riemannian Geometry, volume 103 of Pure and Applied Mathematics. Academic Press Inc. [Harcourt Brace Jovanovich Publishers], New York, 1983.

[SE10] Lennart Simonsson and Lars Eldén. Grassmann algorithms for low rank approximation of matrices with missing values. BIT Numer. Math., 50(1):173-191, 2010. 
[Smi94] Steven T. Smith. Optimization techniques on Riemannian manifolds. In Anthony Bloch, editor, Hamiltonian and gradient flows, algorithms and control, volume 3 of Fields Inst. Commun., pages 113-136. Amer. Math. Soc., Providence, RI, 1994.

[Van11] Bart Vandereycken. Low-rank matrix completion by Riemannian optimization. submitted, 2011. 